\title{
Galileon gravity and its relevance to late time cosmic acceleration
}

\author{
Radouane Gannouji ${ }^{1}$ and M. Sami ${ }^{2}$ \\ ${ }^{1}$ IUCAA, Post Bag 4, Ganeshkhind, Pune 411 00\%, India \\ ${ }^{2}$ Centre of Theoretical Physics, Jamia Millia Islamia, New Delhi-110025, India
}

\begin{abstract}
We consider the covariant galileon gravity taking into account the third order and fourth order scalar field Lagrangians $L_{3}(\pi)$ and $L_{4}(\pi)$ consisting of three and four $\pi$ 's with four and five derivatives acting on them respectively. The background dynamical equations are set up for the system under consideration and the stability of the self accelerating solution is demonstrated in general setting. We extended this study to the general case of the fifth order theory. For spherically symmetric static background, we spell out conditions for suppression of fifth force effects mediated by the galileon field $\pi$. We study field perturbations in the fixed background and investigate conditions for their causal propagation. We also briefly discuss metric fluctuations and derive evolution equation for matter perturbations in galileon gravity.
\end{abstract}

\section{INTRODUCTION}

The phenomenon of late time cosmic acceleration [1-4] is as challenging theoretically as was the problem of black body radiation whose resolution unveiled many secrets of micro physics. At present, there is no definite clue for the theoretical understanding of the nature of cosmic repulsion. In recent years, a variety of approaches have been employed to attack the problem. According to the standard lore, the late time acceleration can be accounted for by supplementing the energy momentum tensor by an exotic fluid component with large negative pressure dubbed dark energy [5, 6] The simplest candidate of dark energy is provided by cosmological constant $\Lambda$. However, its small numerical value leads to fine tuning problem and we do not understand why it becomes important today a la coincidence problem.

Scalar fields provide an interesting alternative to cosmological constant though they do not address the cosmological constant problem. To this effect, cosmological dynamics of a variety of scalar fields has been investigated in the literature(see review [5] for details). They can mimic cosmological constant like behavior at late times and can provide a viable cosmological dynamics at early epochs. Scalar field models with generic features are capable of alleviating the fine tuning and coincidence problems. As for the observation, at present, it is absolutely consistent with $\Lambda$ but at the same time, a large number of scalar field models are also permitted. Future data should allow to narrow down the class of permissible models of dark energy.

It is quite possible that there is no dark energy and the late cosmic acceleration is an artifact of infrared modification of gravity. We know that gravity is modified at short distance and there is no guarantee that it would not suffer any correction at large scales where it is never verified directly. Large scale modifications might arise from extra dimensional effects or can be inspired by fundamental theories. They can also be motivated by phenomenological considerations such as $f(R)$ theories of gravity [7] or the massive theories of gravity. However, any large scale modification of gravity should reconcile with local physics constraints and should have potential of being distinguished from cosmological constant.

The infrared modified theories of gravity essentially contain additional degrees of freedom. The $f(R)$ theories contain a scalar field which mediates fifth force and might contradicts the local gravity constraints such as the solar system or laboratory tests. Broadly, two mechanisms for hiding the scalar field effects locally have been employed in the literature. In $f(R)$ theories of gravity, the scalar field is screened via the so-called chameleon mechanism [8], by making scalar field mass dependent on the local matter density. In generic models of $f(R)$ gravity $[9]$, the chameleon mechanism allows to satisfy the local gravity constraints but at the same time make these models vulnerable to curvature singularity whose resolution requires the fine tuning worse than the one encountered in $\Lambda C D M$ model. The problem can be alleviated by invoking $R^{2}$ correction but the scenario becomes problematic if extended to early universe [9].

An alternative possibility of large scale modification of gravity is provided by an effective scalar field $\pi$ dubbed galileon [10]. In particular such a field appears in the decoupling limit of DGP. The Lagrangian of the field respects the so called shift symmetry in a Minkowskian background: $\pi \rightarrow \pi+c$ and $\partial_{\mu} \pi \rightarrow \partial_{\mu} \pi+b_{\mu}$ where $c$ and $b_{\mu}$ are constants. Thank to this symmetry, the equations of motion for the field contain only second derivatives. In four space time dimensions, there exist five Lagrangians $L_{i}, i=1,5$ where $L_{1}$ is linear in $\pi, L_{2}$ contains normal kinetic term. $L_{3}$ involves three $\pi$ 's and four derivatives acting on them. This Lagrangian is obtained in the decoupling limit of DGP. The fourth and the fifth order Lagrangians involve four $\pi$ 's and six derivatives, five $\pi$ 's and seven derivatives acting on the field respectively. A general covariant form of galileon Lagrangian is obtained in Ref.[11] (see also Ref.[12] on the related theme). 
In DGP or its 4 dimensional generalizations-galileon gravity, the effects of extra degree are suppressed using the Vainshtein [13] mechanism which allows us to recover general relativity at small scales due to non-linear interaction. From this point of view, the DGP model is attractive model which has a self-accelerating solution, an asymptotically de Sitter solution even in the absence of vacuum energy. Unfortunately this solution suffers from instabilities [14 19].

galileon gravity can give rise to late time acceleration and is interesting for the following reasons: (i) It is free from negative energy instabilities. (ii) Unlike $f(R)$ theories, galileon modified gravity does not suffer from curvature singularity. (iii)The chameleon mechanism in $f(R)$ might come into conflict with the equivalence principle if the test bodies are considered as extended whereas the Vainshtein mechanism is free from this problem [20].

In this paper we study 4 th order galileon gravity including $L_{3}$ and $L_{4}$ terms in the Lagrangian. We set up FRW background dynamics and examine the self accelerating solution. We carry out detailed investigations on the stability of the solutions and discuss the spherical symmetric solutions to check the local suppression of $\pi$ effects. We also investigate matter perturbations in the model under consideration.

\section{LOWEST ORDER GALILEON GRAVITY AND ITS SELF ACCELERATING FRW BACKGROUND}

Recently, an interesting generalization of the DGP action in 4D was proposed in Ref. 10]. The authors considered a consistent general action with a self interacting scalar field $(\pi)$ coupled. It is remarkable that the action can be motivated by higher dimensional considerations [21]. In what follows we shall consider the action is invariant under Galilean transformation

$$
\pi(x) \rightarrow \pi(x)+b_{\mu} x^{\mu}+c
$$

For the sake of simplicity, we first examine the galileon model in the lowest non-trivial order keeping up to third order term $L_{3}$ in the Lagrangian,

$$
\mathcal{S}=\int \mathrm{d}^{4} x \sqrt{-g}\left(\frac{R}{2}+c_{1} \pi-\frac{c_{2}}{2}(\nabla \pi)^{2}-\frac{c_{3}}{2}(\nabla \pi)^{2} \square \pi\right)+\mathcal{S}_{m}\left[\psi_{m}, e^{2 \beta \pi} g_{\mu \nu}\right]
$$

Similar expression occurs in the DGP model. The corresponding Einstein's equations are

$$
\begin{aligned}
G_{\mu \nu} & =T_{\mu \nu}^{(m)}+c_{1} \pi g_{\mu \nu}+c_{2}\left(\pi_{; \mu} \pi_{; \nu}-\frac{1}{2} g_{\mu \nu}(\nabla \pi)^{2}\right)+c_{3}\left(\pi_{, \mu} \pi_{; \nu} \square \pi+g_{\mu \nu} \pi_{; \lambda} \pi^{; \lambda \rho} \pi_{; \rho}-\pi^{; \rho}\left[\pi_{; \mu} \pi_{; \nu \rho}+\pi_{; \nu} \pi_{; \mu \rho}\right]\right) \\
0 & =\beta T^{(m)}+c_{1}+c_{2} \square \pi+c_{3}\left((\square \pi)^{2}-\pi_{; \mu \nu} \pi^{; \mu \nu}-R^{\mu \nu} \pi_{; \mu} \pi_{; \nu}\right)
\end{aligned}
$$

where $T^{(m)}$ is the trace of the matter energy-momentum tensor, $T_{\mu \nu}^{(m)} \equiv-(2 / \sqrt{-g}) \times \delta \mathcal{S}_{m} / \delta g^{\mu \nu}$. In spatially flat FRW background Eq.(4) gives rise to the following Friedmann equation

$$
\begin{aligned}
3 H^{2} & =\rho_{m}-c_{1} \pi+\frac{c_{2}}{2} \dot{\pi}^{2}-3 c_{3} H \dot{\pi}^{3} \\
2 \dot{H}+3 H^{2} & =-c_{1} \pi-\frac{c_{2}}{2} \dot{\pi}^{2}-c_{3} \dot{\pi}^{2} \ddot{\pi} \\
\beta \rho_{m} & =c_{1}-c_{2}(3 H \dot{\pi}+\ddot{\pi})+3 c_{3} \dot{\pi}\left(3 H^{2} \dot{\pi}+\dot{H} \dot{\pi}+2 H \ddot{\pi}\right)
\end{aligned}
$$

It is interesting to note that Eq.(17) exhibits a self accelerating solution given by

$$
\begin{aligned}
3 H^{2} & =-c_{1} \pi+\frac{c_{2}}{2} \dot{\pi}^{2}-3 c_{3} H \dot{\pi}^{3} \\
& =-c_{1} \pi-\frac{c_{2}}{2} \dot{\pi}^{2}
\end{aligned}
$$

which means that $c_{1}=0$ (we assume $\dot{\pi} \neq 0$ ) and $H^{4}=-c_{2}^{3} / 54 c_{3}^{2}$. This last condition is impossible to satisfy as $c_{2}$ should be positive for stability of the theory.

We therefore conclude that a stable self accelerating solution, in general, does not exist in the third order galileon gravity with $(\nabla \pi)^{2} \square \pi$ term in the Einstein frame. It is therefore necessary to invoke the higher order terms $L_{4}$ and $L_{5}$. In the discussion, to follow, we shall demonstrate that the desired solution can be obtained by adding the fourth order term in the action (2). The analysis becomes cumbersome in the presence of 5th order term which completes the Lagrangian of galileon gravity. We have included the corresponding discussion and results in the Appendix. 


\section{GENERALIZATION TO NEXT HIGHER ORDER}

Let us consider the full covariant action of galileon gravity $[10,11]$.

$$
\mathcal{S}=\int \mathrm{d}^{4} x \sqrt{-g}\left(\frac{R}{2}+c_{i} L^{(i)}\right)+\mathcal{S}_{m}\left[\psi_{m}, e^{2 \beta \pi} g_{\mu \nu}\right]
$$

where $\left\{c_{i}\right\}$ are constants and the $L_{i}^{\prime s}$ are given by

$$
\begin{aligned}
L^{(1)} & =\pi \\
L^{(2)} & =-\frac{1}{2}(\nabla \pi)^{2} \equiv-\frac{1}{2} \pi_{; \mu} \pi^{; \mu} \\
L^{(3)} & =-\frac{1}{2}(\nabla \pi)^{2} \square \pi \\
L^{(4)} & =-\frac{1}{2}(\nabla \pi)^{2}\left[(\square \pi)^{2}-\pi_{; \mu \nu} \pi^{; \mu \nu}+\pi^{; \mu} \pi^{; \mu} G_{\mu \nu}\right]+(\square \pi) \pi_{; \mu} \pi_{; \nu} \pi^{; \mu \nu}-\pi_{; \mu} \pi^{; \mu \nu} \pi_{; \nu \rho} \pi^{; \rho}
\end{aligned}
$$

Varying the action (10) with respect $\pi$ and the metric $g_{\mu \nu}$, we obtained the field equation for $\pi$ and Einstein equations $^{1}$

$$
\begin{aligned}
c_{i} \mathcal{E}^{(i)} & =-\beta T^{(m)} \\
G_{\mu \nu} & =T_{\mu \nu}^{(m)}+c_{i} T_{\mu \nu}^{(i)}
\end{aligned}
$$

where $\mathcal{E}^{(i)}=(1 / \sqrt{-g}) \times \frac{\delta \mathcal{S}^{(i)}}{\delta \pi}$ and $T_{\mu \nu}^{(i)}=-(2 / \sqrt{-g}) \times \delta \mathcal{S}^{(i)} / \delta g^{\mu \nu}$ with $S^{(i)} \equiv \int \mathrm{d}^{4} x \sqrt{-g} L^{(i)}$ where $\mathcal{E}^{\prime} s$ and $T_{\mu \nu}^{(1)^{\prime} s}$ have the following form

$$
\begin{aligned}
\mathcal{E}^{(1)}= & 1 \\
\mathcal{E}^{(2)}= & \square \pi \\
\mathcal{E}^{(3)}= & (\square \pi)^{2}-\pi_{; \mu \nu} \pi^{; \mu \nu}-R^{\mu \nu} \pi_{; \mu} \pi_{; \nu} \\
\mathcal{E}^{(4)}= & 2(\square \pi)^{3}+4\left(\pi_{; \mu}{ }^{\nu} \pi_{; \nu}{ }^{\rho} \pi_{; \rho}{ }^{\mu}\right)-6(\square \pi)\left(\pi_{; \mu \nu} \pi^{; \mu \nu}\right)-(\square \pi)\left(\pi_{; \mu} \pi^{; \mu}\right) R-2\left(\pi_{; \mu} \pi^{; \mu \nu} \pi_{; \nu}\right) R \\
& -4(\square \pi)\left(\pi_{; \mu} R^{\mu \nu} \pi_{; \nu}\right)+2(\nabla \pi)^{2}\left(\pi_{; \mu \nu} R^{\mu \nu}\right)+8\left(\pi_{; \mu} \pi^{; \mu \nu} R_{\nu \rho} \pi^{; \rho}\right)+4\left(\pi_{; \mu} \pi_{; \nu} \pi_{; \rho \sigma} R^{\mu \rho \nu \sigma}\right)
\end{aligned}
$$

$$
\begin{aligned}
T_{\mu \nu}^{(1)}= & \pi_{\mu \nu} \\
T_{\mu \nu}^{(2)}= & \pi_{; \mu} \pi_{; \nu}-\frac{1}{2} g_{\mu \nu}(\nabla \pi)^{2} \\
T_{\mu \nu}^{(3)}= & \pi_{, \mu} \pi_{; \nu} \square \pi+g_{\mu \nu} \pi_{; \lambda} \pi^{; \lambda \rho} \pi_{; \rho}-\pi^{; \rho}\left[\pi_{; \mu} \pi_{; \nu \rho}+\pi_{; \nu} \pi_{; \mu \rho}\right] \\
T_{\mu \nu}^{(4)}= & -4(\square \pi) \pi^{; \rho}\left[\pi_{; \mu} \pi_{; \rho \nu}+\pi_{; \nu} \pi_{; \rho \mu}\right]+2(\square \pi)^{2}\left(\pi_{; \mu} \pi_{; \nu}\right)-2(\square \pi)(\nabla \pi)^{2}\left(\pi_{; \mu \nu}\right)-4\left(\pi_{; \lambda} \pi^{; \lambda \rho} \pi_{; \rho}\right)\left(\pi_{; \mu \nu}\right) \\
& +4\left(\pi^{; \lambda} \pi_{; \lambda \mu}\right)\left(\pi^{; \rho} \pi_{; \rho \nu}\right)-2\left(\pi_{; \lambda \rho} \pi^{; \lambda \rho}\right)\left(\pi_{; \mu} \pi_{; \nu}\right)+2(\nabla \pi)^{2}\left(\pi_{; \mu}^{; \rho} \pi_{; \rho \nu}\right)+4 \pi_{; \lambda} \pi^{; \lambda \rho}\left[\pi_{; \rho \mu} \pi_{; \nu}+\pi_{; \rho \nu} \pi_{; \mu}\right] \\
& +(\square \pi)^{2}(\nabla \pi)^{2} g_{\mu \nu}+4(\square \pi)\left(\pi_{; \lambda} \pi^{; \lambda \rho} \pi_{; \rho}\right) g_{\mu \nu}-4\left(\pi_{; \lambda} \pi^{; \lambda \rho} \pi_{; \rho \sigma} \pi^{; \sigma}\right) g_{\mu \nu}-(\nabla \pi)^{2}\left(\pi_{; \rho \sigma} \pi^{; \rho \sigma}\right) g_{\mu \nu} \\
& -(\nabla \pi)^{2}\left(\pi_{; \mu} \pi_{; \nu}\right) R+\frac{1}{4}(\nabla \pi)^{4} g_{\mu \nu} R+2(\nabla \pi)^{2} \pi^{; \rho}\left[R_{\rho \mu} \pi_{; \nu}+R_{\rho \nu} \pi_{; \mu}\right]-\frac{1}{2}(\nabla \pi)^{4} R_{\mu \nu} \\
& -2(\nabla \pi)^{2}\left(\pi_{; \rho} R^{\rho \sigma} \pi_{; \sigma}\right) g_{\mu \nu}+2(\nabla \pi)^{2}\left(\pi^{; \rho} \pi^{; \sigma} R_{\mu \rho \nu \sigma}\right)
\end{aligned}
$$

It may be instructive to define the effective energy density and pressure for $\pi$ matter. Indeed, for each $(i)$,

${ }^{1}$ We have $T_{\mu \nu}^{(4)}=-T_{\mu \nu}^{\prime(4)}$ and $\mathcal{E}^{(4)}=-\frac{1}{2} \mathcal{E}^{\prime(4)}$ compared to [1]. 


$$
\nabla^{\mu} T_{\mu \nu}^{(i)}=\pi_{; \nu} \mathcal{E}^{(i)}
$$

which allows us to write the equation of conservation

$$
\nabla^{\mu} T_{\mu \nu}^{(m)}=\beta T^{(m)} \pi_{; \nu}
$$

For each $(i)$, assuming the perfect fluid form, we can express the field energy momentum tensor as, $T_{\mu \nu}^{(i)}=$ $\left(\rho^{(i)}+P^{(i)}\right) u_{\mu} u_{\nu}+P^{(i)} g_{\mu \nu}$ with $u_{\mu} \equiv-\sigma \frac{\pi_{; \mu}}{\sqrt{-(\nabla \pi)^{2}}}$ and $\sigma=\operatorname{sign}\left(\pi_{; 0}\right)$. The corresponding expressions for $\rho^{i}$ and $P^{i}$ have following form

$$
\begin{aligned}
& \rho^{(1)}=-\pi \\
& \rho^{(2)}=-\frac{1}{2}(\nabla \pi)^{2} \\
& P^{(1)}=\pi \\
& \rho^{(3)}=\pi_{; \lambda} \pi^{; \lambda \rho} \pi_{; \rho}-(\nabla \pi)^{2} \square \pi \\
& P^{(2)}=-\frac{1}{2}(\nabla \pi)^{2} \\
& \rho^{(4)}=6 \square \pi \pi_{; \lambda} \pi^{; \lambda \rho} \pi_{; \rho}-3(\square \pi)^{2}(\nabla \pi)^{2}+3(\nabla \pi)^{2} \\
& +\frac{3}{4} R(\nabla \pi)^{4}-\frac{3}{2}(\nabla \pi)^{2} \pi^{; \rho} R_{\rho \sigma} \pi^{; \sigma} \\
& -2 \pi^{; \rho} \pi^{; \sigma} \pi^{; \mu} \pi^{; \nu} R_{\mu \rho \nu \sigma}-6 \pi_{; \lambda} \pi^{; \lambda \rho} \pi_{; \rho \sigma} \pi^{; \sigma} \\
& P^{(3)}=\pi_{; \lambda} \pi^{; \lambda \rho} \pi_{; \rho} \\
& P^{(4)}=(\square \pi)^{2}(\nabla \pi)^{2}+4 \square \pi_{; \lambda} \pi^{; \lambda \rho} \pi_{; \rho}-4 \pi_{; \lambda} \pi^{; \lambda \rho} \pi_{; \rho \sigma} \pi^{; \sigma} \\
& -(\nabla \pi)^{2} \pi_{; \rho \sigma} \pi^{; \rho \sigma}+\frac{1}{4} R(\nabla \pi)^{4}-2(\nabla \pi)^{2} \pi^{; \rho} R_{\rho \sigma} \pi^{; \sigma}
\end{aligned}
$$

In the following section, we shall analyze the background solution of the fourth order theory.

\section{BACKGROUND DYNAMICS}

Assuming the spatially flat background, we obtain evolution equations of the fourth order galileon cosmology,

$$
\begin{aligned}
3 H^{2} & =\rho_{m}+\frac{c_{2}}{2} \dot{\pi}^{2}-3 c_{3} H \dot{\pi}^{3}+\frac{45}{2} c_{4} H^{2} \dot{\pi}^{4} \\
2 \dot{H}+3 H^{2} & =-\frac{c_{2}}{2} \dot{\pi}^{2}-c_{3} \dot{\pi}^{2} \ddot{\pi}+\frac{3}{2} c_{4} \dot{\pi}^{3}\left(3 H^{2} \dot{\pi}+2 \dot{H} \dot{\pi}+8 H \ddot{\pi}\right) \\
\beta \rho_{m} & =-c_{2}(3 H \dot{\pi}+\ddot{\pi})+3 c_{3} \dot{\pi}\left(3 H^{2} \dot{\pi}+\dot{H} \dot{\pi}+2 H \ddot{\pi}\right)-18 c_{4} H \dot{\pi}^{2}\left(3 H^{2} \dot{\pi}+2 \dot{H} \dot{\pi}+3 H \ddot{\pi}\right),
\end{aligned}
$$

where we have assumed, $c_{1}=0$ as we do not want include the cosmological constant explicitly. In this case, the conservation has standard form in presence of coupling $\beta$

$$
\dot{\rho}_{m}+3 H \rho_{m}=\beta \rho_{m} \dot{\pi}
$$

We may also define the total energy density and pressure for the scalar field $\pi$

$$
\begin{aligned}
& \rho_{\pi}=\frac{c_{2}}{2} \dot{\pi}^{2}-3 c_{3} H \dot{\pi}^{3}+\frac{45}{2} c_{4} H^{2} \dot{\pi}^{4} \\
& P_{\pi}=\frac{c_{2}}{2} \dot{\pi}^{2}+c_{3} \dot{\pi}^{2} \ddot{\pi}-\frac{3}{2} c_{4} \dot{\pi}^{3}\left(3 H^{2} \dot{\pi}+2 \dot{H} \dot{\pi}+8 H \ddot{\pi}\right)
\end{aligned}
$$

which can be used to check for the total equation of state parameter $w_{\pi}=P_{\pi} / \rho_{\pi}$. In the next section, we discuss the self accelerating solution of galileon cosmology.

\section{SELF ACCELERATING SOLUTION}

A self acceleration solution is characterized by $\rho_{m}=0$ and $H \equiv H_{0}=C^{s t}$.

In this case, using equation (27), we find that $\dot{\pi} \equiv \dot{\pi}_{0}=C^{s t}$ and

$$
\begin{aligned}
H_{0} \dot{\pi}_{0}^{ \pm} & =\frac{c_{3} \pm \sqrt{c_{3}^{2}-8 c_{2} c_{4}}}{12 c_{4}} \\
48 H_{0}^{2} & =\left(\dot{\pi}_{0}^{ \pm}\right)^{2} A_{ \pm}
\end{aligned}
$$


With $A_{ \pm}=\frac{c_{3}^{2}-12 c_{2} c_{4} \pm c_{3} \sqrt{c_{3}^{2}-8 c_{2} c_{4}}}{c_{4}}$.

The existence of the self accelerating solution then implies the following conditions on constants $c_{1}, c_{2}, c_{3}$ and $c_{4}$,

$$
\begin{aligned}
c_{3}^{2}-8 c_{2} c_{4}>0 \\
A_{+}>0 \text { or } A_{-}>0
\end{aligned}
$$

It is not difficult to check the stability of the solution. Let us consider the perturbation of the two self-accelerating solutions,

$$
H=H_{0}+\delta H, \quad \dot{\pi}=\dot{\pi}_{0}+\delta \dot{\pi}
$$

It can easily be checked that $\dot{H}=-3 H_{0} \delta H$, which means that the self-accelerating solutions are stable.

\section{SPHERICALLY SYMMETRIC SOLUTION}

We shall now be interested in the spherically symmetric static solution . We consider a static point-like source of mass $M$, located at the origin: $T^{(m)}=-M \delta^{3}(\vec{x})$ and look for a spherically symmetric static solution for the field $\pi(r)$ described by the following differential equation

$$
\frac{c_{2}}{r^{2}} \frac{\mathrm{d}}{\mathrm{d} r}\left[r^{2} \pi^{\prime}(r)\right]+2 \frac{c_{3}}{r^{2}} \frac{\mathrm{d}}{\mathrm{d} r}\left[r \pi^{\prime}(r)^{2}\right]+4 \frac{c_{4}}{r^{2}} \frac{\mathrm{d}}{\mathrm{d} r}\left[\pi^{\prime}(r)^{3}\right]=\beta M \delta^{3}(\vec{x})
$$

Integration of Eq.(38) gives the following relation,

$$
c_{2}\left(\frac{\pi^{\prime}(r)}{r}\right)+2 c_{3}\left(\frac{\pi^{\prime}(r)}{r}\right)^{2}+4 c_{4}\left(\frac{\pi^{\prime}(r)}{r}\right)^{3}=\beta \frac{r_{s}}{r^{3}}
$$

Where $r_{s}$ is the Schwarzschild radius of the source.

The conditions of existence of the solution are derived following Ref. [10]:

if $\beta>0 \Rightarrow \operatorname{sign}\left(c_{2}\right)=\operatorname{sign}\left(c_{4}\right)$ and $c_{3}>-\sqrt{3 c_{2} c_{4}}$ which means that $c_{3}>\sqrt{8 c_{2} c_{4}}$ if we consider the condition (35)

if $\beta<0 \Rightarrow \operatorname{sign}\left(c_{2}\right)=\operatorname{sign}\left(c_{4}\right)$ and $c_{3}<\sqrt{3 c_{2} c_{4}}$ which means that $c_{3}<-\sqrt{8 c_{2} c_{4}}$ if we consider the condition (35)

In case $\beta<0$, at short distances, the solution is not analytic in the neighborhood of $r=0$ and we shall not consider this case any further.

Whereas for $\beta>0$,

$$
\pi^{\prime}(r)=\frac{\left(c_{4}^{2} r_{s} \beta\right)^{1 / 3}}{2^{2 / 3} c_{4}}
$$

Then the galileon-mediated force is suppressed compared to the gravitational force:

$$
\frac{F_{\pi}}{F_{\text {grav }}}=\left(\frac{r}{r_{\star}}\right)^{2} \ll 1, \quad \text { with } \quad r_{\star}^{3}=\left(\frac{\left|c_{4}\right|}{2 \beta}\right)^{1 / 2} r_{s}
$$

At large distances, we have

$$
\frac{F_{\pi}}{F_{\text {grav }}}=2 \frac{\beta}{c_{2}}
$$

If $\beta \simeq c_{2}$, the galileon field can lead to late time acceleration of universe. 


\section{STABILITY}

In order to study the stability of the aforesaid static solutions, we perturb the scalar field $\pi: \pi \rightarrow \pi+\phi$ in a fixed metric $g_{\mu \nu}$. We have neglected the perturbations of the metric induced by the perturbations of the scalar field $\phi$; the method is referred to test field approximation.

In order to proceed with the test field approximation, let us rewrite the quadratic term in $\phi$ in the action

$$
\mathcal{S}_{\phi}=\int \sqrt{-g} \mathrm{~d}^{4} x c^{i} Z_{(i)}^{\mu \nu} \phi_{; \mu} \phi_{; \nu}
$$

with

$$
\begin{aligned}
Z_{(1)}^{\mu \nu}= & 0 \\
Z_{(2)}^{\mu \nu}= & -\frac{1}{2} g^{\mu \nu} \\
Z_{(3)}^{\mu \nu}= & \pi^{; \mu \nu}-g^{\mu \nu} \square \pi \\
Z_{(4)}^{\mu \nu}= & -2 \pi^{; \mu} R^{\nu \rho} \pi_{; \rho}-2 \pi^{; \nu} R^{\mu \rho} \pi_{; \rho}-R^{\mu \nu}(\nabla \pi)^{2}+R \pi^{; \mu} \pi^{; \nu}+6 \square \pi \pi^{; \mu \nu}-6 \pi^{; \mu \rho} \pi_{; \rho}^{\nu}+2 R^{\mu \rho \sigma \nu} \pi_{; \rho} \pi_{; \sigma} \\
& +g^{\mu \nu}\left(3 \pi_{; \rho \sigma} \pi^{; \rho \sigma}-3(\square \pi)^{2}+2 R_{\rho \sigma} \pi^{; \rho} \pi^{; \sigma}+\frac{1}{2} R(\nabla \pi)^{2}\right)
\end{aligned}
$$

The equation of motion for perturbations that follow from action (43) is

$$
-2 c^{i} Z_{(i)}^{\mu \nu} \phi_{; \mu \nu}-2 c^{i} Z_{; \mu}^{\mu \nu} \phi_{; \nu}+8 \beta^{2} \phi T^{(m)}=0,
$$

which we shall use in the subsequent sections.

\section{A. Cauchy-problem}

Following the theorem due to Leray [22], the scalar field $\phi$ propagates causally in the effective metric $G_{\text {eff }}^{\mu \nu}=-2 c^{i} Z_{(i)}^{\mu \nu}$ if spacetime $\left(\mathcal{M}, G_{\text {eff }}^{\mu \nu}\right)$ is globally hyperbolic. A necessary condition but not sufficient is the requirement of the hyperbolicity of the equation (49) that is a Lorentzian signature of the effective metric $G_{\mathrm{eff}}^{\mu \nu}$.

For the static spherical solution, the hyperbolicity is defined by

$$
\begin{aligned}
c_{2}+2 c_{3}\left(2 \pi^{\prime} / r+\pi^{\prime \prime}\right)+12 c_{4}\left(\pi^{\prime} / r+2 \pi^{\prime \prime}\right) \pi^{\prime} / r & >0 \\
c_{2}+4 c_{3} \pi^{\prime} / r+12 c_{4}\left(\pi^{\prime} / r\right)^{2} & >0 \\
c_{2}+2 c_{3}\left(\pi^{\prime} / r+\pi^{\prime \prime}\right)+12 c_{4} \pi^{\prime \prime} \pi^{\prime} / r & >0
\end{aligned}
$$

At large distances, we obtain the following conditions

$$
\begin{gathered}
c_{2}-36 \beta^{2} \frac{c_{4}}{c_{2}^{2}} \frac{r_{s}^{2}}{r^{6}}>0 \\
c_{2}+4 \beta \frac{c_{3}}{c_{2}} \frac{r_{s}}{r^{3}}>0 \\
c_{2}-2 \beta \frac{c_{3}}{c_{2}} \frac{r_{s}}{r^{3}}>0
\end{gathered}
$$

which reduce to $c_{2}>0$ at very large scales.

At small distances, we need to impose the conditions, $c_{4}>0$ and $c_{3}>0$.

For the de Sitter phase, the hyperbolicity is defined by 


$$
\begin{aligned}
G_{\mathrm{eff}}^{00} & =-\frac{1}{4}\left(A_{ \pm}+4 c_{2}\right)<0, \\
a^{2} G_{\mathrm{eff}}^{11} & =\frac{1}{36}\left(A_{ \pm}-4 c_{2}\right)>0
\end{aligned}
$$

which implies that $A_{ \pm}>4 c_{2}$.

We should however emphasize that this solution is derived when the scalar field is dominant (de Sitter phase), therefore any small perturbation of the scalar field leads to a perturbation of the metric and the test field approximation is then no longer true.

\section{B. Hamiltonian approach}

An alternative way to study the stability is related to the positive definiteness of Hamiltonian of the underlying theory. In a locally inertial frame, the Hamiltonian is

$$
\mathcal{H}=-\frac{1}{2} G_{\mathrm{eff}}^{00} \dot{\phi}^{2}+\frac{1}{2} G_{\mathrm{eff}}^{k l} \phi_{, k} \phi_{, l}
$$

The condition of hyperbolicity of equation (49) is sufficient for the Hamiltonian to be bounded from below. The condition of hyperbolicity imposes an important restriction on sound speed which we consider next.

\section{Speed of sound}

From the equation (49), it is obvious to define the "sound speed" $c_{s}^{2}$; the condition of hyperbolicity of the equation restricts $c_{s}$ to real values $c_{s}^{2}>0$. It is straightforward to see that the condition of $c_{s}$ to be real, restrict the signature of the effective metric to $(-,+,+,+)$ or $(+,-,-,-)$.

However, if we also impose the positivity of the Hamiltonian, we have to consider the effective metric with the same signature that as that of the original metric $g_{\mu \nu}$ which is $(-,+,+,+)$, in our case . This condition for non superluminal behavior of the scalar field $\phi$ is expressed by $c_{s}^{2}<1$.

In case of the de Sitter phase, it is trivial to see that $c_{s}^{2}=\frac{A_{ \pm}-4 c_{2}}{9\left(A_{ \pm}+4 c_{2}\right)}<1$ (because of the conditions of stability of the theory $\left(c_{2}>0\right.$ and $\left.\left.A_{ \pm}>0\right)\right)$. But the problem is more delicate for the spherically symmetric solution. Indeed, equation (49) can be rewritten as

$$
G_{\mathrm{eff}}^{00} \ddot{\phi}+G_{\mathrm{eff}}^{11} \partial_{r}^{2} \phi+G_{\mathrm{eff}}^{22} r^{2} \partial_{\Omega}^{2} \phi+\text { first derivatives of } \phi+\ldots=0
$$

where $\partial_{\Omega}^{2}$ is the angular part of the Laplacian.

Therefore we can define the speed of radial and angular excitations as follows,

$$
\begin{aligned}
c_{r}^{2} & =-\frac{G_{\mathrm{eff}}^{11}}{G_{\mathrm{eff}}^{00}}=\frac{c_{2}+4 c_{3} \pi^{\prime} / r+12 c_{4} \pi^{\prime 2} / r^{2}}{c_{2}+2 c_{3}\left(2 \pi^{\prime} / r+\pi^{\prime \prime}\right)+12 c_{4}\left(\pi^{\prime 2} / r^{2}+2 \pi^{\prime \prime} \pi^{\prime} / r\right)} \\
c_{\Omega}^{2} & =-\frac{r^{2} G_{\mathrm{eff}}^{22}}{G_{\mathrm{eff}}^{00}}=\frac{c_{2}+2 c_{3}\left(\pi^{\prime} / r+\pi^{\prime \prime}\right)+12 c_{4} \pi^{\prime \prime} \pi^{\prime} / r}{c_{2}+2 c_{3}\left(2 \pi^{\prime} / r+\pi^{\prime \prime}\right)+12 c_{4}\left(\pi^{\prime 2} / r^{2}+2 \pi^{\prime \prime} \pi^{\prime} / r\right)},
\end{aligned}
$$

which at large distances gives rise to

$$
\begin{aligned}
& c_{r}^{2} \approx 1+4 \beta \frac{c_{3}}{c_{2}^{2}} \frac{r_{s}}{r^{3}} \\
& c_{\Omega}^{2} \approx 1-2 \beta \frac{c_{3}}{c_{2}^{2}} \frac{r_{s}}{r^{3}}
\end{aligned}
$$

whereas for small distances, we find 


$$
\begin{aligned}
c_{r}^{2} & =1 \\
c_{\Omega}^{2} & \approx \frac{c_{3}}{6 c_{4}} \frac{r}{\pi^{\prime}}
\end{aligned}
$$

It is clear that at large distances, we have a superluminal behavior $\left(c_{r}^{2}>1\right)$ of the scalar field $\phi$ for the static spherically solution, but this behavior is physically possible if the theory does not have Closed Causal Curves (CCCs) which leads to paradoxes [22, 23]. It is known that if a spacetime is stably causal, it does not possesses CCCs which means that a global time can be defined. This is the case if we can define a global time for the two metrics $g_{\mu \nu}$ and $G_{\mu \nu}$.

For the static spherically symmetric solution, we will consider the Minkowsky time $\eta^{\mu \nu} \nabla_{\mu} t \nabla_{\nu} t=-1$.

Then

$$
G_{e f f}^{\mu \nu} \nabla_{\mu} t \nabla_{\nu} t=-c_{2}-2 c_{3}\left(2 \pi^{\prime} / r+\pi^{\prime \prime}\right)-12 c_{4}\left(\pi^{\prime} / r+2 \pi^{\prime \prime}\right) \pi^{\prime} / r
$$

Eq.(66), at large distances, reduces to

$$
G_{e f f}^{\mu \nu} \nabla_{\mu} t \nabla_{\nu} t=-c_{2}+36 \beta^{2} \frac{c_{4}}{c_{2}^{2}} \frac{r_{s}^{2}}{r^{6}}
$$

which is negative iff $r^{6}>36 \beta^{2} r_{s}^{2} c_{4} / c_{2}^{3}$.

If this condition is satisfied then the space time $\left(M, G_{\mu \nu}^{\mathrm{eff}}\right)$ is stably causal which means that no closed timelike curves exist. We should emphasize that this condition is satisfied if the equation (49) is hyperbolic.

\section{METRIC PERTURBATIONS}

Let us consider the perturbed FLRW spacetime with scalar metric perturbations in the longitudinal gauge

$$
\mathrm{d} s^{2}=-(1+2 \phi) \mathrm{d} t^{2}+a^{2}(1-2 \psi) \mathrm{d} x^{2}
$$

The linear matter perturbations $\delta_{m}$ on super horizon scales satisfy the evolution equation similar to the one in Einstein gravity

$$
\ddot{\delta}_{m}+2 H \dot{\delta}_{m}-\frac{G_{e f f}}{2} \rho_{m} \delta_{m}=0
$$

with the modified Newtonian constant,

$$
G_{e f f}=1+\frac{2\left(c_{3} \dot{\pi}^{2}+2 \beta\right)^{2}+c_{4} N_{4}}{4 c_{2}-2 c_{3}^{2} \dot{\pi}^{4}-16 c_{3} H \dot{\pi}-8 c_{3} \ddot{\pi}+c_{4} D_{4}}
$$

where $N_{4}$ and $D_{4}$ are given by

$$
\begin{aligned}
N_{4} & =14 c_{2} \dot{\pi}^{4}+c_{3}^{2} \dot{\pi}^{8}-88 c_{3} H \dot{\pi}^{5}+4 c_{3} \beta \dot{\pi}^{6}+20 c_{3} \dot{\pi}^{4} \ddot{\pi}-64 H \beta \dot{\pi}^{3}-24 \beta^{2} \dot{\pi}^{4}+96 \beta \dot{\pi}^{2} \ddot{\pi} \\
& +c_{4}\left(-9 c_{2} \dot{\pi}^{8}-12 c_{3} H \dot{\pi}^{9}-54 c_{3} \dot{\pi}^{8} \ddot{\pi}+492 H^{2} \dot{\pi}^{6}-96 H \beta \dot{\pi}^{7}-48 H \dot{\pi}^{5} \ddot{\pi}+168 \dot{H} \dot{\pi}^{6}+18 \beta^{2} \dot{\pi}^{8}-144 \beta \dot{\pi}^{6} \ddot{\pi}+288 \dot{\pi}^{4} \ddot{\pi}^{2}\right) \\
& +c_{4}^{2}\left(18\left(11 H^{2}-6 \dot{H}\right) \dot{\pi}^{10}+648 H \dot{\pi}^{9} \ddot{\pi}\right) \\
D_{4} & =-12 c_{2} \dot{\pi}^{4}-c_{3}^{2} \dot{\pi}^{8}+80 c_{3} H \dot{\pi}^{5}-24 c_{3} \dot{\pi}^{4} \ddot{\pi}+8\left(13 H^{2}+6 \dot{H}\right) \dot{\pi}^{2}+96 H \dot{\pi} \ddot{\pi} \\
& +c_{4}\left(9 c_{2} \dot{\pi}^{8}+12 c_{3} H \dot{\pi}^{9}+54 c_{3} \dot{\pi}^{8} \ddot{\pi}-24\left(17 H^{2}+6 \dot{H}\right) \dot{\pi}^{6}+288 H \dot{\pi}^{5} \ddot{\pi}\right) \\
& +c_{4}^{2}\left(18\left(6 \dot{H}-11 H^{2}\right) \dot{\pi}^{10}-648 H \dot{\pi}^{9} \ddot{\pi}\right)
\end{aligned}
$$

The study of generic models of modified gravity shows that there is a characteristic signature in the growth function $f=\frac{\mathrm{d} \ln \delta_{\mathrm{m}}}{\mathrm{d} \ln \mathrm{a}}$ which can allow us to distinguish these models from $\Lambda C D M$ and other dynamical dark energy models within the frame work of Einstein gravity. We expect similar features in galileon gravity. We shall address this important issue in our future work. 


\section{CONCLUSION}

In this paper, we have investigated galileon gravity in its general form. The model consists of an effective field $\pi$ Lagrangian consisting of five terms $\sum_{1}^{5} c_{i} L^{i}$ added to Einstein-Hilbert action such that the field equation are of second order. In spatially flat FRW background, we set up the evolutions equations in the model and examine the existence and stability of self accelerating solutions. We point out that these solutions, in general $(\dot{\pi} \neq 0)$, are not stable in the third order galileon theory. We extend the analysis to the fourth and fifth order theory. In fourth order theory, self accelerating solutions exist provided that $c_{3}^{2}-8 c_{2} c_{4}>0$ and $A_{+}>0$ or $A_{-}>0$. We show that there is at least one stable self-accelerating solution in this case. The analysis is cumbersome in case of 5 th order theory and we have included the corresponding results in the appendix. The conclusions reached in fourth order galileon theory are shown to hold in general. In case of the spherically symmetric static solution, we find that the solution exists provided that $c_{3}>\sqrt{8 c_{2} c_{4}}$. The solution is stable and the fifth force can lead the acceleration of the universe if we assume $\beta \simeq c_{2}$ and and $c_{4}>0$. We find as expected that the galileon force mediated by the scalar field $\pi$ is negligibly small at small scales, because of the non-linear terms in the Lagrangian. However, the fifth force is of the order of the gravitational force at large scales in case, $\beta \simeq c_{2}$.

Subsequently, we investigated the stability issues associated with the spherically symmetric solution. Using the fixed background method, we found superluminal behavior of perturbations as was noticed in [10]. It is really interesting that despite the superluminal behavior, there exist static solutions which do not possess any Closed Causal Curve allowing to avoid paradoxes related to micro-causality and making the solution physically acceptable. The model has a well posed Cauchy problem and no Closed Causal Curves exists in this model even if we have a superluminal behavior of the perturbation of the scalar field in the static spherically symmetric situation at large distances.

We have included brief discussion on the metric perturbations and have set up the evolution equation for linear

matter perturbation in the galileon gravity. In our opinion, it is important to study the growth function $f=\frac{\mathrm{d} \ln \delta_{\mathrm{m}}}{\mathrm{d} \ln \mathrm{a}}$ which can provide a discriminating signature of galileon gravity; we defer this analysis to our future work.

\section{ACKNOWLEDGEMENTS}

We thank S. Deser for a useful comment. MS is supported by DST project No.SR/S2/HEP-002/2008. R. G. thanks CTP, Jamia Millia Islamia for hospitality where this work was carried out.

[1] S. Perlmutter et al. [Supernova Cosmology Project Collaboration], Astrophys. J. 517 (1999) 565 arXiv:astro-ph/9812133.

[2] A. G. Riess et al. [Supernova Search Team Collaboration], Astron. J. 116 (1998) 1009 arXiv:astro-ph/9805201.

[3] D. N. Spergel et al. [WMAP Collaboration], Astrophys. J. Suppl. 170 (2007) 377 arXiv:astro-ph/0603449].

[4] U. Seljak et al. [SDSS Collaboration], Phys. Rev. D 71 (2005) 103515 arXiv:astro-ph/0407372.

[5] E. J. Copeland, M. Sami and S. Tsujikawa, Int.J.Mod.Phys.D 15, 1753 (2006).

[6] V. Sahni and A. A. Starobinsky, Int.J.Mod.Phys.D 9, 373 (2000); T. Padmanabhan, Phys.Rep. 380, 235 (2003); E. V. Linder, astro-ph/0704.2064; J. Frieman, M. Turner and D. Huterer, arXiv:0803.0982; R. Caldwell and M. Kamionkowski, arXiv:0903.0866; A. Silvestri and M. Trodden, arXiv:0904.0024; M. Sami, arXiv:0904.3445. P. J. E. Peebles and B. Ratra, Rev. Mod. Phys. 75, 559 (2003); L. Perivolaropoulos, astro-ph/0601014, N. Straumann, arXiv:gr-qc/0311083; J. Frieman, arXiv:0904.1832 M. Sami, Lect. Notes Phys.72, 219(2007); M. Sami, arXiv:0901.0756, R. Bean, arXiv:1003.4468, M. Sami, arXiv:0904.3445; Shinji Tsujikawa, arXiv:1004.1493

[7] S. Capozziello, Int. J. Mod. Phys. D 11, 483 (2002);

S. Capozziello, S. Carloni, and A. Troisi, arXiv:astro-ph/0303041

S. Carroll, V. Duvvuri, M. Trodden, and M. Turner, Phys. Rev. D 70 (2004) 043528; T. P. Sotiriou and V. Faraoni, arXiv:0805.1726, S. Nojiri and S. Odintsov, Gen. Rel. Grav. 36 (2004) 1765 arXiv:hep-th/0308176; Antonio De Felice, Shinji Tsujikawa, arXiv:1002.4928.

[8] D. F. Mota and J. D. Barrow, Phys. Lett. B 581 (2004) 141 arXiv:astro-ph/0306047; J. Khoury and A. Weltman, Phys. Rev. Lett. 93 (2004) 171104 arXiv:astro-ph/0309300.

[9] W. Hu and I. Sawicki, Phys. Rev. D 76 (2007) 064004 arXiv:0705.1158 [astro-ph]]; A. A. Starobinsky, JETP Lett. 86 (2007) 157; M. Sami, S. Rai Choudhury, Phys.Rev.D80:127501,2009 I. Thongkool, M. Sami, R. Gannouji and S. Jhingan, Phys. Rev. D 80 (2009) 043523 arXiv:0906.2460 [hep-th]]; S. A. Appleby and R. A. Battye, arXiv:0803.1081 A. V. Frolov, arXiv:0803.2500; M Abdalla, S nojiri and S D Odintsov, hep-th/0409177; K Bamba, S Nojiri and S D Odintsov, arXiv:0807.2575 hep-th]; S Nojiri and S D Odintsov, arXiv:0804.3519 hep-th].

[10] A. Nicolis, R. Rattazzi and E. Trincherini, Phys. Rev. D 79 (2009) 064036 arXiv:0811.2197 [hep-th]].

[11] C. Deffayet, G. Esposito-Farese and A. Vikman, Phys. Rev. D 79 (2009) 084003 arXiv:0901.1314 [hep-th]]; C. Deffayet, S. Deser and G. Esposito-Farese, Phys. Rev. D 80 (2009) 064015 [arXiv:0906.1967 [gr-qc]] 
[12] N. Chow and J. Khoury, Phys. Rev. D 80 (2009) 024037 arXiv:0905.1325 [hep-th]]; F. P. Silva and K. Koyama, Phys. Rev. D 80 (2009) 121301 arXiv:0909.4538 [astro-ph.CO]]; T. Kobayashi, arXiv:1003.3281 [astro-ph.CO]; T. Kobayashi, H. Tashiro and D. Suzuki, Phys. Rev. D 81 (2010) 063513 arXiv:0912.4641] [astro-ph.CO]].

[13] A. I. Vainshtein, Phys. Lett. B 39 (1972) 393.

[14] M. A. Luty, M. Porrati and R. Rattazzi, JHEP 0309 (2003) 029 arXiv:hep-th/0303116.

[15] A. Nicolis and R. Rattazzi, JHEP 0406 (2004) 059 arXiv:hep-th/0404159.

[16] D. Gorbunov, K. Koyama and S. Sibiryakov, Phys. Rev. D 73 (2006) 044016 arXiv:hep-th/0512097.

[17] C. Charmousis, R. Gregory, N. Kaloper and A. Padilla, JHEP 0610 (2006) 066 arXiv:hep-th/0604086].

[18] G. Dvali, G. Gabadadze, O. Pujolas and R. Rahman, Phys. Rev. D 75 (2007) 124013 arXiv:hep-th/0612016].

[19] R. Gregory, N. Kaloper, R. C. Myers and A. Padilla, JHEP 0710 (2007) 069 arXiv:0707.2666 [hep-th]].

[20] L. Hui, A. Nicolis and C. Stubbs, Phys. Rev. D 80 (2009) 104002 [arXiv:0905.2966 [astro-ph.CO]].

[21] C. de Rham and A. J. Tolley, arXiv:1003.5917 [hep-th].

[22] R. M. Wald, Chicago, Usa: Univ. Pr. (1984) $491 p$

[23] E. Babichev, V. Mukhanov and A. Vikman, JHEP 0802 (2008) 101 arXiv:0708.0561 [hep-th]].

\section{Appendix A: The full Lagrangian of galileon gravity: Extension of the model to the fifth order term, $L^{(5)}$}

We consider the term $L^{(5)}$ derived in [11]

$$
\begin{aligned}
L^{(5)}= & -\frac{1}{2}(\nabla \pi)^{2}\left[(\square \pi)^{3}-3(\square \pi)\left(\pi_{; \mu \nu} \pi^{; \mu \nu}\right)+2\left(\pi_{; \mu}{ }^{\nu} \pi_{; \nu}{ }^{\rho} \pi_{; \rho}{ }^{\mu}\right)-3\left(\pi_{; \mu} \pi_{; \nu} \pi_{; \rho \sigma} R^{\mu \rho \nu \sigma}\right)\right. \\
& \left.-18\left(\pi_{; \nu} \pi^{; \nu \rho} R_{\rho \sigma} \pi^{; \sigma}\right)+3(\square \pi)\left(\pi_{; \nu} R^{\nu \rho} \pi_{; \rho}\right)+\frac{15}{2}(\nabla \pi)^{2}\left(\pi_{; \nu} \pi^{; \nu \rho} \pi_{; \rho}\right) R\right] \\
& +3\left[\pi_{; \mu} \pi^{; \mu \nu} \pi_{; \nu \rho} \pi^{; \rho \lambda} \pi_{; \lambda}-(\square \pi)\left(\pi_{; \mu} \pi^{; \mu \nu} \pi_{; \nu \rho} \pi^{; \rho}\right)\right] \\
& +\frac{3}{2}\left[(\square \pi)^{2}\left(\pi_{; \mu} \pi^{; \mu \nu} \pi_{; \nu}\right)-\left(\pi_{; \mu \nu} \pi^{; \mu \nu}\right)\left(\pi_{; \rho} \pi^{; \rho \lambda} \pi_{; \lambda}\right)\right]
\end{aligned}
$$

then the equations (15]16) are modified by

$$
\begin{aligned}
& \mathcal{E}^{(5)}=\frac{5}{2}(\square \pi)^{4}-15(\square \pi)^{2}\left(\pi_{; \mu \nu} \pi^{; \mu \nu}\right)-\frac{15}{4}(\square \pi)^{2}(\nabla \pi)^{2} R-\frac{15}{2}(\square \pi)^{2}\left(\pi_{; \mu} R^{\mu \nu} \pi_{; \nu}\right) \\
& +20(\square \pi)\left(\pi_{; \mu}{ }^{\nu} \pi_{; \nu}{ }^{\rho} \pi_{; \rho}{ }^{\mu}\right)-\frac{15}{2}(\square \pi)\left(\pi_{; \mu} \pi^{; \mu \nu} \pi_{; \nu}\right) R+15(\square \pi)(\nabla \pi)^{2}\left(\pi_{; \nu \rho} R^{\nu \rho}\right) \\
& +30(\square \pi)\left(\pi_{; \mu} \pi^{; \mu \nu} R_{\nu \rho} \pi^{; \rho}\right)+15(\square \pi)\left(\pi_{; \mu} \pi_{; \nu} \pi_{; \rho \sigma} R^{\mu \rho \nu \sigma}\right)+\frac{15}{2}\left(\pi_{; \mu \nu} \pi^{; \mu \nu}\right)^{2}-15\left(\pi_{; \mu \nu} \pi^{; \nu \rho} \pi_{; \rho \sigma} \pi^{; \sigma \mu}\right) \\
& +\frac{15}{4}(\nabla \pi)^{2}\left(\pi_{; \nu \rho} \pi^{; \nu \rho}\right) R+\frac{15}{2}\left(\pi_{; \mu} \pi^{; \mu \nu} \pi_{; \nu \rho} \pi^{; \rho}\right) R+\frac{15}{2}\left(\pi_{; \mu \nu} \pi^{; \mu \nu}\right)\left(\pi_{; \rho} R^{\rho \sigma} \pi_{; \sigma}\right) \\
& +15\left(\pi_{; \mu} \pi^{; \mu \nu} \pi_{; \nu}\right)\left(\pi_{; \rho \sigma} R^{\rho \sigma}\right)-15(\nabla \pi)^{2}\left(\pi_{; \nu}{ }^{\rho} R_{\rho}{ }^{\sigma} \pi_{; \sigma}{ }^{\nu}\right)-30\left(\pi_{; \mu} \pi^{; \mu \nu} \pi_{; \nu \rho} R^{\rho \sigma} \pi_{; \sigma}\right) \\
& -15\left(\pi_{; \mu} \pi^{; \mu \nu} R_{\nu \rho} \pi^{; \rho \sigma} \pi_{; \sigma}\right)-\frac{15}{2}(\nabla \pi)^{2}\left(\pi_{; \nu \rho} \pi_{; \sigma \lambda} R^{\nu \sigma \rho \lambda}\right)-15\left(\pi_{; \mu} \pi_{; \nu} \pi_{; \rho \sigma} \pi_{\lambda}^{; \sigma} R^{\mu \rho \nu \lambda}\right) \\
& +30\left(\pi_{; \lambda} \pi_{\mu}^{; \lambda} \pi_{; \nu \rho} \pi_{; \sigma} R^{\mu \nu \rho \sigma}\right)+\frac{15}{4}(\nabla \pi)^{2}\left(\pi_{; \nu} R^{\nu \rho} \pi_{; \rho}\right) R-\frac{15}{2}(\nabla \pi)^{2}\left(\pi_{; \nu} R^{\nu \rho} R_{\rho \sigma} \pi^{; \sigma}\right) \\
& -\frac{15}{2}(\nabla \pi)^{2}\left(\pi_{; \nu} \pi_{; \rho} R_{\sigma \lambda} R^{\nu \sigma \rho \lambda}\right)+\frac{15}{4}(\nabla \pi)^{2}\left(\pi_{; \nu} \pi_{; \rho} R_{\sigma \kappa \lambda}^{\nu} R^{\rho \sigma \kappa \lambda}\right) \\
& T_{\mu \nu}^{(5)}=\frac{5}{2}(\square \pi)^{3}\left(\pi_{; \mu} \pi_{; \nu}\right)+\frac{5}{2}(\square \pi)^{3}(\nabla \pi)^{2} g_{\mu \nu}-\frac{15}{2}(\square \pi)^{2}(\nabla \pi)^{2}\left(\pi_{; \mu \nu}\right)-\frac{15}{2}(\square \pi)^{2} \pi^{; \rho}\left[\pi_{; \rho \mu} \pi_{; \nu}+\pi_{; \rho \nu} \pi_{; \mu}\right] \\
& +\frac{15}{2}(\square \pi)^{2}\left(\pi_{; \rho} \pi^{; \rho \sigma} \pi_{; \sigma}\right) g_{\mu \nu}+15(\square \pi)(\nabla \pi)^{2}\left(\pi_{; \mu \sigma} \pi_{\nu}^{; \sigma}\right)-15(\square \pi)\left(\pi_{; \rho} \pi^{; \rho \sigma} \pi_{; \sigma}\right)\left(\pi_{; \mu \nu}\right) \\
& -\frac{15}{2}(\square \pi)\left(\pi_{; \rho \sigma} \pi^{; \rho \sigma}\right)\left(\pi_{; \mu} \pi_{; \nu}\right)+15(\square \pi)\left(\pi^{; \rho} \pi_{; \rho \mu}\right)\left(\pi^{; \sigma} \pi_{; \sigma \nu}\right)+15(\square \pi) \pi_{; \rho} \pi^{; \rho \sigma}\left[\pi_{; \sigma \mu} \pi_{; \nu}+\pi_{; \sigma \nu} \pi_{; \mu}\right] \\
& -\frac{15}{2}(\square \pi)(\nabla \pi)^{2}\left(\pi_{; \sigma \lambda} \pi^{; \sigma \lambda}\right) g_{\mu \nu}-15(\square \pi)\left(\pi_{; \rho} \pi^{; \rho \sigma} \pi_{; \sigma \lambda} \pi^{; \lambda}\right) g_{\mu \nu}-\frac{15}{4}(\square \pi)(\nabla \pi)^{2}\left(\pi_{; \mu} \pi_{; \nu}\right) R \\
& +\frac{15}{2}(\square \pi)(\nabla \pi)^{2} \pi^{; \sigma}\left[R_{\sigma \mu} \pi_{; \nu}+R_{\sigma \nu} \pi_{; \mu}\right]-\frac{15}{2}(\square \pi)(\nabla \pi)^{2}\left(\pi_{; \sigma} R^{\sigma \lambda} \pi_{; \lambda}\right) g_{\mu \nu}
\end{aligned}
$$




$$
\begin{aligned}
& +\frac{15}{2}(\square \pi)(\nabla \pi)^{2}\left(\pi^{; \sigma} \pi^{; \lambda} R_{\mu \sigma \nu \lambda}\right)+\frac{15}{2}(\nabla \pi)^{2}\left(\pi_{; \sigma \lambda} \pi^{; \sigma \lambda}\right)\left(\pi_{; \mu \nu}\right)-15(\nabla \pi)^{2}\left(\pi_{; \mu \sigma} \pi^{; \sigma \lambda} \pi_{; \lambda \nu}\right) \\
& +15\left(\pi_{; \rho} \pi^{; \rho \sigma} \pi_{; \sigma}\right)\left(\pi_{; \mu \lambda} \pi_{\nu}^{; \lambda}\right)+15\left(\pi_{; \rho} \pi^{; \rho \sigma} \pi_{; \sigma \lambda} \pi^{; \lambda}\right)\left(\pi_{; \mu \nu}\right)+5\left(\pi_{; \rho}{ }^{\sigma} \pi_{; \sigma}{ }^{\lambda} \pi_{; \lambda}{ }^{\rho}\right)\left(\pi_{; \mu} \pi_{; \nu}\right) \\
& +\frac{15}{2}\left(\pi_{; \sigma \lambda} \pi^{; \sigma \lambda}\right) \pi^{; \rho}\left[\pi_{; \rho \mu} \pi_{; \nu}+\pi_{; \rho \nu} \pi_{; \mu}\right]-15 \pi^{; \rho} \pi_{; \rho \sigma} \pi^{; \sigma \lambda}\left[\pi_{; \lambda \mu} \pi_{; \nu}+\pi_{; \lambda \nu} \pi_{; \mu}\right] \\
& -15 \pi_{; \rho} \pi^{; \rho \lambda} \pi^{; \sigma}\left[\pi_{; \lambda \mu} \pi_{; \sigma \nu}+\pi_{; \lambda \nu} \pi_{; \sigma \mu}\right]+5(\nabla \pi)^{2}\left(\pi_{; \sigma}{ }^{\lambda} \pi_{; \lambda}{ }^{\kappa} \pi_{; \kappa}{ }^{\sigma}\right) g_{\mu \nu}-\frac{15}{2}\left(\pi_{; \rho} \pi^{; \rho \sigma} \pi_{; \sigma}\right)\left(\pi_{; \lambda \kappa} \pi^{; \lambda \kappa}\right) g_{\mu \nu} \\
& +15\left(\pi_{; \rho} \pi^{; \rho \sigma} \pi_{; \sigma \lambda} \pi^{; \lambda \kappa} \pi_{; \kappa}\right) g_{\mu \nu}+\frac{15}{4}(\nabla \pi)^{2} \pi^{; \sigma}\left[\pi_{; \sigma \mu} \pi_{; \nu}+\pi_{; \sigma \nu} \pi_{; \mu}\right] R-\frac{15}{4}(\nabla \pi)^{2}\left(\pi_{; \sigma} \pi^{; \sigma \lambda} \pi_{; \lambda}\right) R g_{\mu \nu} \\
& +\frac{15}{2}(\nabla \pi)^{2}\left(\pi_{; \sigma} \pi^{; \sigma \lambda} \pi_{; \lambda}\right) R_{\mu \nu}+\frac{15}{2}(\nabla \pi)^{2}\left(\pi_{; \sigma} R^{\sigma \lambda} \pi_{; \lambda}\right)\left(\pi_{; \mu \nu}\right)+\frac{15}{2}(\nabla \pi)^{2}\left(\pi_{; \sigma \lambda} R^{\sigma \lambda}\right)\left(\pi_{; \mu} \pi_{; \nu}\right) \\
& -\frac{15}{2}(\nabla \pi)^{2} \pi_{; \sigma} \pi^{; \sigma \lambda}\left[R_{\lambda \mu} \pi_{; \nu}+R_{\lambda \nu} \pi_{; \mu}\right]-\frac{15}{2}(\nabla \pi)^{2} \pi^{; \lambda} \pi^{; \sigma}\left[R_{\lambda \mu} \pi_{; \sigma \nu}+R_{\lambda \nu} \pi_{; \sigma \mu}\right] \\
& -\frac{15}{2}(\nabla \pi)^{2} \pi_{; \sigma} R^{\sigma \lambda}\left[\pi_{; \lambda \mu} \pi_{; \nu}+\pi_{; \lambda \nu} \pi_{; \mu}\right]+15(\nabla \pi)^{2}\left(\pi_{; \sigma} \pi^{; \sigma \lambda} R_{\lambda \kappa} \pi^{; \kappa}\right) g_{\mu \nu} \\
& -\frac{15}{2}(\nabla \pi)^{2} \pi^{; \sigma} \pi^{; \lambda \kappa}\left[R_{\mu \lambda \sigma \kappa} \pi_{; \nu}+R_{\nu \lambda \sigma \kappa} \pi_{; \mu}\right]+\frac{15}{2}(\nabla \pi)^{2} \pi^{; \sigma} \pi^{; \lambda}\left[R_{\mu \sigma \lambda \kappa} \pi_{\nu}^{; \kappa}+R_{\nu \sigma \lambda \kappa} \pi_{\mu}\right] \\
& -\frac{15}{2}(\nabla \pi)^{2} \pi_{; \sigma} \pi^{; \sigma \lambda} \pi^{; \kappa}\left[R_{\mu \lambda \nu \kappa}+R_{\nu \lambda \mu \kappa}\right]+\frac{15}{2}(\nabla \pi)^{2}\left(\pi_{; \sigma} \pi_{; \lambda} \pi_{; \kappa \tau} R^{\sigma \kappa \lambda \tau}\right) g_{\mu \nu .}
\end{aligned}
$$

The Friedmann equations for this model are

$$
\begin{aligned}
3 H^{2}= & \rho_{m}+\frac{c_{2}}{2} \dot{\pi}^{2}-3 c_{3} H \dot{\pi}^{3}+\frac{45}{2} c_{4} H^{2} \dot{\pi}^{4}-\frac{105}{2} c_{5} H^{3} \dot{\pi}^{5} \\
2 \dot{H}+3 H^{2}= & -\frac{c_{2}}{2} \dot{\pi}^{2}-c_{3} \dot{\pi}^{2} \ddot{\pi}+\frac{3}{2} c_{4} \dot{\pi}^{3}\left(3 H^{2} \dot{\pi}+2 \dot{H} \dot{\pi}+8 H \ddot{\pi}\right)-\frac{15}{2} c_{5} H_{\pi^{4}}\left(2 H^{2} \dot{\pi}+2 \dot{H} \dot{\pi}+5 H \ddot{\pi}\right) \\
\beta \rho_{m}= & -c_{2}(3 H \dot{\pi}+\ddot{\pi})+3 c_{3} \dot{\pi}\left(3 H^{2} \dot{\pi}+\dot{H} \dot{\pi}+2 H \ddot{\pi}\right)-18 c_{4} H \dot{\pi}^{2}\left(3 H^{2} \dot{\pi}+2 \dot{H} \dot{\pi}+3 H \ddot{\pi}\right) \\
& +\frac{75}{2} c_{5} H^{2} \dot{\pi}^{3}\left(3 H^{2} \dot{\pi}+3 \dot{H} \dot{\pi}+4 H \ddot{\pi}\right)
\end{aligned}
$$

Therefore the self accelerating solution exist if there is a real solution of the equation

$$
\begin{aligned}
c_{2}-3 c_{3} X+18 c_{4} X^{2}-\frac{75}{2} c_{5} X^{3} & =0 \\
c_{2}-9 c_{4} X^{2}+30 c_{5} X^{3} & <0, \text { with } X=H_{0} \dot{\pi}_{0}
\end{aligned}
$$

If we have a solution of this system therefore the self accelerating solution is stable. In fact if we consider a perturbation of the self accelerating solution $H=H_{0}+\delta H$ and $\dot{\pi}=\dot{\pi}_{0}+\delta \dot{\pi}$, it is straightforward to see that, $\delta \dot{H}=-3 H_{0} \delta H$.

We found that the spherical symmetric solution is not modified by the fifth term as it was noticed in Ref. [10]. 